\title{
Impact of Fibrinogen Levels on Angiographic Progression and 12-Year Survival in the Armed Forces Regression Study
}

\author{
Ganesh P. Devendra, BA,' Stephen A. Hart, BS,' Edwin J. Whitney, MD, ${ }^{2}$ and \\ Richard A. Krasuski, MD'
}

\begin{abstract}
We assessed the role of fibrinogen levels on angiographic progression and long-term survival among III patients with coronary disease enrolled in the Armed Forces Regression Study (AFREGS). Baseline fibrinogen levels and quantitative coronary angiography were performed initially and at 30 months. Progression or nonregression of coronary disease was more prevalent in patients with high fibrinogen than patients with normal fibrinogen $(66.1 \%$ vs $45.5 \% ; P=.022)$. Twelve-year cardiovascular $(\mathrm{CV})$ mortality was substantially higher if fibrinogen was elevated $(17.9 \%$ vs $3.6 \%, P=.016)$. Among patients with elevated fibrinogen and angiographic progression or nonregression, there were 10 deaths and all were $\mathrm{CV}$. Elevated levels of fibrinogen predict the angiographic progression of existing coronary disease and likelihood of CV death. Among patients with elevated levels of fibrinogen, angiographic progression identifies a significantly increased likelihood of a fatal CV event.
\end{abstract}

\section{Keywords}

fibrinogen, atherosclerosis, angiographic progression

\section{Introduction}

Inflammation appears central to the initiation and progression of atheromatous disease. ${ }^{1}$ Accordingly, several inflammatory markers have been evaluated as potential predictors of cardiovascular (CV) risk. ${ }^{2-4}$ Among the potential predictors, plasma fibrinogen is a particularly attractive target as it is an acute phase reactant, a determinant of blood viscosity, a participant in thrombogenesis, and a cofactor for platelet aggregation. ${ }^{5}$ As such, it has long been hypothesized that elevated plasma fibrinogen may reflect a heightened inflammatory state within the vascular wall that is pathogenic for atherosclerosis. ${ }^{6}$ This is further supported by the observation that elevated plasma fibrinogen is an independent risk factor for coronary heart disease (CHD), stroke, and vascular death ${ }^{7,8}$ and is also a strong predictor of myocardial infarction in patients experiencing angina pectoris. ${ }^{9}$ In addition to its predictive value in $\mathrm{CV}$ disease, fibrinogen independently correlates with atherosclerotic burden. ${ }^{10}$ This unique property suggests that fibrinogen is not only a binary predictor of risk but may correlate with the progression of atheromatous disease.

Several potential mechanisms have attempted to explain fibrinogen's role in atheromatous disease. However, it is unclear whether fibrinogen is a causative or reactive agent in the inflammatory process. ${ }^{11}$ It has been suggested that fibrinogen acts as a chemotactic agent that recruits smooth muscle cells from the media into the intima, thereby promoting the development of atheroma. ${ }^{12,13}$ It has also been suggested that during atherogenesis, an inflamed vascular wall induces the production of cytokines, which will in turn upregulate the hepatic production of acute phase reactants such as fibrinogen. ${ }^{6}$ Further complicating this issue are epidemiological studies that have shown equivocal results regarding fibrinogen as an independent $\mathrm{CV}$ risk factor. ${ }^{14,15}$ Given this disparity in understanding, further clarification of the complex interaction between inflammation, angiographic progression, and mortality is needed.

The Armed Forces Regression Study (AFREGS) ${ }^{16}$ was a randomized, double-blind, placebo-controlled trial of aggressive medical therapy targeted to increase high-density lipoprotein (HDL) on a baseline of aggressive dietary and lifestyle intervention. The purpose of this substudy was to examine the ability of baseline plasma fibrinogen to predict (1) angiographic progression of atherosclerosis and (2) long-term

\footnotetext{
' Division of Clinical Cardiology, Department of CV Medicine, Cleveland Clinic Foundation, Cleveland, $\mathrm{OH}$, USA

${ }^{2}$ Heart and Vascular Institute of San Antonio, San Antonio, TX, US
}

Corresponding Author:

Richard A. Krasuski, Department of CV Medicine, Desk J2-4, Cleveland Clinic Foundation, 9500 Euclid Ave, Cleveland, OH 44I95, USA.

Email: krasusr@ccf.org 
survival among patients with angiographically evident coronary disease.

\section{Methods}

Men and women aged $<76$ years, with suspected stable CHD, were recruited from within a 150-mile radius of Wilford Hall Medical Center in San Antonio, Texas. Each patient was required to have a measurable stenosis between $30 \%$ and $80 \%$ of the luminal diameter within the coronary tree by quantitative coronary angiography. A measure of global coronary atherosclerosis was obtained by averaging the percentage of stenosis in each diseased segment. This was done at baseline and repeated at 30-months follow-up; the catheterization techniques have been previously described in detail. ${ }^{16}$ Patients with greater than an $80 \%$ stenosis of a single vessel were eligible only if they had a favorable prognosis based on functional testing (ability to exercise for more than 9 minutes on a full-Bruce Protocol exercise treadmill test).

The exclusions have previously been published $^{16}$ and included a major vascular event (myocardial infarction, cerebrovascular accident, coronary artery bypass grafting, or other coronary catheter-based intervention) within 6 months, a history of congestive heart failure (other than in the setting of a myocardial infarction), a left ventricular ejection fraction $<40 \%$ by ventriculography, or diabetes (fasting blood glucose $\geq 110 \mathrm{mg} / \mathrm{dL}$ ).

Our subpopulation of 111 AFREGS patients was defined in the following manner: of the 143 patients in the original AFREGS analysis, 128 completed their 30-month follow-up catheterization. Of these 128 , only 111 patients had baseline fasting fibrinogen levels (measured by the Clauss clotting assay). ${ }^{17}$ Patients were thoroughly informed of the details of the study and voluntarily enrolled between January of 1993 and March of 1994. The initial cohort was followed until September of 1996 when the database was locked. Because of funding issues, full analysis of the study data as well as the subsequent long-term follow-up did not occur until 2004, when the amended institutional review board (IRB) application was approved and the initial results were published. Patient follow-up for this substudy occurred for a total of $12.0 \pm 0.2$ years and patients were monitored for all-cause death and $\mathrm{CV}$ death. No patients were lost to follow-up and cause of death was confirmed by hospital record or death certificate if available. Each patient initially signed an "Informed Consent Statement" that was reviewed and approved by the Institutional Review Board at Wilford Hall Air Force Medical Center in San Antonio, Texas. Patient selection has been previously described in detail. ${ }^{16}$

We compiled and analyzed data by using JMP 8.0 software (SAS Institute, Inc, Cary, North Carolina). We dichotomized the patients into "high" and "normal" fibrinogen based on the median baseline fibrinogen of $286 \mathrm{mg} / \mathrm{dL}$, with the high fibrinogen group having a baseline fibrinogen of $\geq 286 \mathrm{mg} / \mathrm{dL}$. Dichotomized outcomes were compared by using the chisquare test or Fischer exact test where appropriate. Continuous variables were compared using a 2-tailed $t$ test. Multivariate modeling was performed using forward and backward stepwise nominal logistic regression with a parameter estimate inclusion cutoff of $P=.2$ to determine a subset of baseline parameters to be used in the model. The baseline variables included in the first step were age, systolic blood pressure, total cholesterol, LDL, HDL, triglycerides, fibrinogen, body mass index (BMI), and fasting blood sugar. Effect likelihood ratios were used to determine effect significance. Survival curves were compared by the log-rank test. For all tests, a $P$ value of .05 or less was considered significant.

\section{Results}

Baseline characteristics of all 111 patients are included in Table 1. The mean fibrinogen for all patients was $293 \pm$ $67 \mathrm{mg} / \mathrm{dL}$, with a median fibrinogen of $286 \mathrm{mg} / \mathrm{dL}$ (interquartile range $=243-330$ ). We chose to separate our patients into high and normal fibrinogen groups based on this median, and this resulted in a mean fibrinogen of $242 \pm 27 \mathrm{mg} / \mathrm{dL}$ in the normal fibrinogen group and $343 \pm 56$ in the high fibrinogen group. This comparison is similar to the means used to define lower fibrinogen $(250 \mathrm{mg} / \mathrm{dL})$ and upper fibrinogen $(350 \mathrm{mg} / \mathrm{dL})$ in a previous meta-analysis. ${ }^{18}$ Fifty-five patients were included in the normal fibrinogen group, whereas 56 patients were included in the high fibrinogen group. Because these patients are a subset of patients from the AFREGS trial, they were randomly assigned to either the treatment group (gemfibrozil $600 \mathrm{mg}$ twice daily, Niacin starting at $250 \mathrm{mg}$ and titrated up to $3000 \mathrm{mg} /$ day as tolerated, and cholestyramine $16 \mathrm{~g} / \mathrm{d}$ ) or placebo therapy for 30 months. Within the normal fibrinogen group, $31(56.4 \%)$ patients received treatment, whereas $28(50.5 \%)$ of patients in the high fibrinogen group received treatment $(P=.57)$. All patients were subject to aggressive diet and exercise modification as previously described. ${ }^{16}$

Table 2 shows the results of a logistic regression analysis of baseline parameters as predictors of death. The 3 most significant variables were age at enrollment, fibrinogen, and triglycerides. Only baseline fibrinogen and age at enrollment had a significance effect $<.05$ ( $P=.02$ and $P=.01$, respectively). Table 3 shows angiographic changes, death, and CV death dichotomized by high versus normal fibrinogen. Over the 30month observation period, $62(55.9 \%)$ patients had progression or no regression of their atherosclerosis. Within the normal fibrinogen group, 25 (45.5\%) patients had angiographic progression or no regression, whereas $37(66.1 \%)$ patients in the high fibrinogen group had progression or no regression $(P=$ $.02)$. Over the 12 -year observation period, there were 17 overall deaths (15.3\%) and $12 \mathrm{CV}$ deaths (10.8\%). Among the overall deaths, $4(7.3 \%)$ were in the normal fibrinogen group and 13 $(23.2 \%)$ in the high fibrinogen group $(P=.02)$. Among the $\mathrm{CV}$ deaths, $2(3.6 \%)$ were in the normal fibrinogen group and $10(17.9 \%)$ in the high fibrinogen group $(P=.02)$. In Table 4 , all-cause mortality and CV mortality are shown dichotomized by those patients with both elevated fibrinogen and 
Table I. Baseline Demographic Data of Patients

\begin{tabular}{|c|c|c|c|c|}
\hline Demographics & All Patients; $\mathrm{n}=\mathrm{I} \mathrm{I} \mathrm{I}$ & $\begin{array}{l}\text { Normal Fibrinogen } \\
\text { (<Median); } \mathrm{n}=55\end{array}$ & $\begin{array}{l}\text { High Fibrinogen } \\
\text { ( } \geq \text { Median); } \mathrm{n}=56\end{array}$ & $P$ Value \\
\hline Age (years; mean $\pm \mathrm{SD}$ ) & $63.4 \pm 6.6$ & $61.9 \pm 6.6$ & $64.9 \pm 6.3$ & .02 \\
\hline History of hypertension (\%) & 72.9 & 74.6 & 71.4 & .83 \\
\hline History of angina (\%) & 64.8 & 58.2 & 71.4 & .17 \\
\hline History of myocardial infarction (\%) & 43.2 & 43.6 & 42.9 & 1.00 \\
\hline Weight (lbs; mean \pm SD) & $182.5 \pm 27.9$ & $182.6 \pm 25.7$ & $182.3 \pm 30.1$ & .96 \\
\hline Body mass index $\left(\mathrm{kg} / \mathrm{m}^{2} ;\right.$ mean $\left.\pm \mathrm{SD}\right)$ & $26.5 \pm 3.6$ & $26.3 \pm 2.9$ & $26.7 \pm 4.2$ & .48 \\
\hline Systolic blood pressured $(\mathrm{mm} \mathrm{Hg}$; mean $\pm \mathrm{SD})$ & $138.2 \pm 15.4$ & $139.9 \pm 13.3$ & $136.6 \pm 17.3$ & .27 \\
\hline Fasting blood glucose $(\mathrm{mg} / \mathrm{dL}$; mean $\pm \mathrm{SD})$ & $81 \pm 13$ & $81 \pm 15$ & $81 \pm 11$ & .79 \\
\hline Total cholesterol (mg/dL; mean $\pm \mathrm{SD})$ & $195 \pm 32$ & $193 \pm 32$ & $197 \pm 32$ & .54 \\
\hline $\mathrm{LDL}(\mathrm{mg} / \mathrm{dL} ;$ mean $\pm \mathrm{SD})$ & $127 \pm 28$ & $127 \pm 28$ & $128 \pm 27$ & .90 \\
\hline Fibrinogen (mg/dl; median) & 286 & & & \\
\hline
\end{tabular}

${ }^{a}$ Treated patients received a regimen of gemfibrozil, niacin, and cholestyramine as part of the AFREGS (Armed Forces Regression Study; Citation) Trial.

angiographic progression or no regression versus patients with elevated fibrinogen and angiographic regression. Among patients with elevated fibrinogen and angiographic progression, there were $10(27 \%)$ all-cause deaths while there were 3 $(15.8 \%)$ all-cause deaths in patients with high fibrinogen and angiographic regression $(P=.28)$. All 10 of the deaths in the patients with high fibrinogen and angiographic progression or no regression were due to $\mathrm{CV}$ causes, whereas none of the 3 deaths in the high fibrinogen and angiographic regression group were due to $\mathrm{CV}$ causes $(P=.01)$. These 3 non-CV deaths were comprised of 1 patient who died from complications of pneumonia and 2 patients who died from lung cancer. Figure 1 compares 12-year survival between the high fibrinogen group and the normal fibrinogen group via Kaplan-Meier analysis. Long-term survival was significantly better in the normal fibrinogen group $(P=.02)$. Of the 107 patients that had repeat fibrinogen levels at 30 months, $34(31.8 \%)$ experienced a fibrinogen reduction that was independent of treatment $(P=$ $.83)$. When patients with fibrinogen increase were compared with those with fibrinogen decrease, there was no significant difference in overall death $(P=.23), \mathrm{CV}$ death $(P=.72)$, or atherosclerotic progression $(P=.67)$.

\section{Discussion}

In patients with seemingly stable atherosclerosis, plasma fibrinogen levels were strongly predictive of long-term clinical outcome. More interestingly, it appeared to predict both CV and non-CV deaths. Although prior studies have noted the longterm impact of fibrinogen elevation on $\mathrm{CV}$ outcome, ${ }^{7}$ on the development of new CV disease, ${ }^{19}$ and on the severity of coronary disease, ${ }^{20}$ this is to our knowledge the first study that
Table 2. Nominal Regression for Baseline Parameters as Predictors of Death

\begin{tabular}{llll}
\hline Baseline Parameter & Odds Ratio & $95 \% \mathrm{Cl}$ & $P$ Value \\
\hline Age at enrollment & 1.14 & $1.04-1.13$ & .01 \\
Fibrinogen & 1.01 & $1.0-1.02$ & .02 \\
Triglycerides & 1.01 & $0.99-1.02$ & .06 \\
\hline
\end{tabular}

demonstrates the implication of elevated fibrinogen on the angiographic progression of existing CHD.

As part of the protocol, all patients underwent angiography at a 30 -month interval; $45.5 \%$ of patients in the normal fibrinogen group had either progression or no regression of coronary disease, whereas $66.1 \%$ had progression or no regression of coronary disease in the high fibrinogen group. These angiographic findings are consistent with the intravascular ultrasound findings where fibrinogen levels were positively correlated with annual changes in plaque cross-sectional area. ${ }^{21}$ This association between fibrinogen levels and progression of coronary disease, as well as the previous finding that fibrinogen levels reflect atherosclerotic burden, ${ }^{10}$ suggests that fibrinogen is more than just a binary predictor of CV mortality and may directly reflect vascular wall inflammation that is part of the process of ongoing atherosclerosis.

In addition to progression of atherosclerosis, elevated fibrinogen predicted both all-cause and CV mortality. During the 12-year observation period, $23.2 \%$ of high fibrinogen patients died, whereas only $7.3 \%$ of low fibrinogen patients died. Moreover, in the high fibrinogen group, $17.9 \%$ of patients suffered a $\mathrm{CV}$ death, whereas as $3.6 \%$ of the normal fibrinogen group suffered a CV death. This is certainly consistent with the notion that fibrinogen is not only a risk factor for CHD but is also a predictor for the sequelae of $\mathrm{CV}$ disease. ${ }^{7,22}$ The predictive 
Table 3. Angiographic Progression and Clinical Events by Fibrinogen

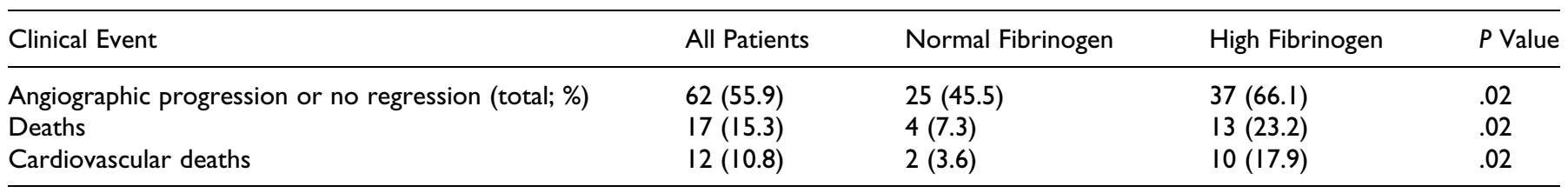

Table 4. Clinical Events by Fibrinogen Level and Angiographic Progression

\begin{tabular}{llll}
\hline & $\begin{array}{l}\text { High Fibrinogen } \\
\text { With Regression } \\
(\mathrm{n}=19)\end{array}$ & $\begin{array}{l}\text { High Fibrinogen + Pro- } \\
\text { gression/Nonregression } \\
(\mathrm{n}=37)\end{array}$ & $\begin{array}{l}P \\
\text { Value }\end{array}$ \\
\hline $\begin{array}{l}\text { Death (total) } \\
(\%)\end{array}$ & $3(15.8)$ & $10(27)$ & .28 \\
$\begin{array}{l}\text { Cardiovascular } \\
\text { death }\end{array}$ & $0(0)$ & $10(27)$ & .01 \\
\hline
\end{tabular}

power of fibrinogen was also demonstrated by our multivariate analysis which showed that only fibrinogen levels and age were significant predictors of mortality. Kaplan-Meier analysis also showed improved survival in the normal fibrinogen group compared with the high fibrinogen group.

The combination of elevated fibrinogen and angiographic progression or nonregression was also highly predictive of CV mortality. There were 10 deaths among this cohort, and all 10 of these deaths were due to $\mathrm{CV}$ causes. In patients with elevated fibrinogen and angiographic regression, there were 3 deaths and none were due to $\mathrm{CV}$ causes. Interestingly, patients who died in the elevated fibrinogen and angiographic regression group died from either cancer or serious infection and, therefore, may have had mechanisms for elevation of acute phase reactants that were separate from CHD. This notion is supported by the finding in a 2005-meta-analysis that fibrinogen is associated with nonvascular causes of death, chiefly comprised of cancer. ${ }^{7}$ Interestingly, there was no significant difference in fibrinogen change between those patients receiving lipid modifying therapy and those receiving placebo. This may not be surprising given that treatment with at least one of the agents studied, gemfibrozil, has previously been shown not to affect fibrinogen levels. ${ }^{23}$

In summary, these data support the notion that fibrinogen is an independent risk factor for both all-cause mortality and CV death. This is also, to our knowledge, the first demonstration that elevated baseline fibrinogen predicts angiographic progression of existing CHD. Furthermore, among patients with elevated baseline fibrinogen levels, angiographic progression identifies a significantly increased likelihood of fatal CV event. Although our study size was limited, our analysis is strengthened by our $100 \%$ retention of participants, our ability to observe long-term outcomes (12 years), and the fact that we could verify cause of death by either hospital record or death certificate. The strong association of elevated fibrinogen with angiographic progression as well as death and CV death explains the impact of inflammation on the type of mortality

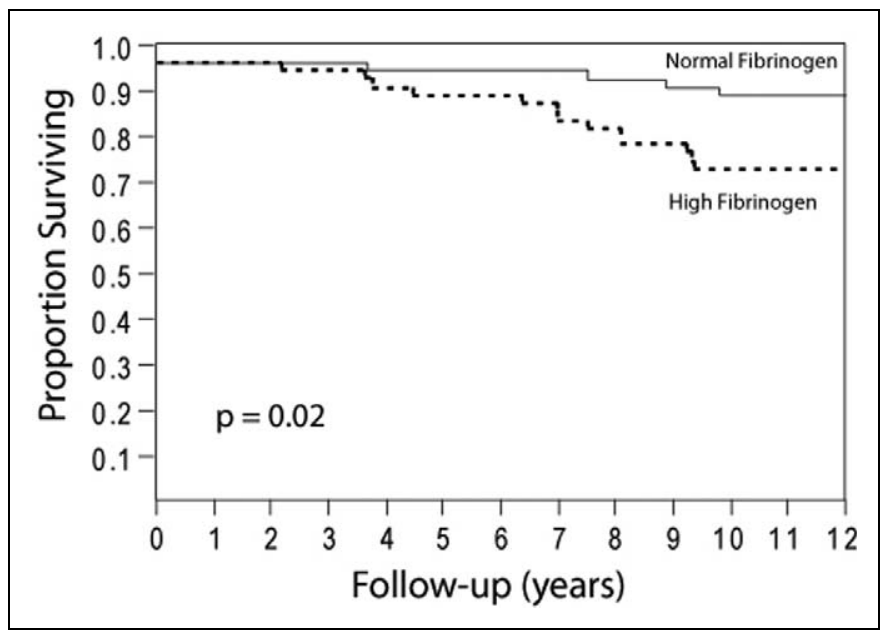

Figure I. Kaplan-Meier survival curves for patients with normal and high baseline fibrinogen.

and attempts to explain why this occurs. Given that the assay for fibrinogen is considered simple and cost-effective, ${ }^{24}$ these findings should encourage the use of fibrinogen as a marker for vascular wall inflammation and underlying atheromatous disease to identify a high-risk population that may benefit from closer observation and possibly pharmacologic intervention.

\section{Conclusions}

Elevated levels of fibrinogen predict the angiographic progression of existing CHD in patients with stable disease. Furthermore, elevated fibrinogen predicted the likelihood of all-cause death and CV death in these patients. Among patients with elevated levels of fibrinogen, concomitant angiographic progression identifies a significantly increased likelihood of a fatal CV event, whereas fibrinogen elevation without angiographic progression appears to predict death from non-CV causation such as cancer or infection.

\section{Declaration of Conflicting Interests}

The authors declared a potential conflict of interest as follows: Dr Krasuski is on the speaker's bureau of Pfizer Pharmaceuticals.

\section{Funding}

Dr Krasuski is on the speaker's bureau of Pfizer Pharmaceuticals, Roche Pharmaceuticals, AGA medical, and United Therapeutics, and is both a consultant for and on the speaker's bureau of Actelion Pharmaceuticals. 


\section{References}

1. Ross R. Atherosclerosis - an inflammatory disease. $N$ Engl $J$ Med. 1999;340(24):115-126.

2. Ballantyne CM, Nambi V. Markers of inflammation and their clinical significance. Atheroscler Suppl. 2005;6(2):21-29.

3. Danesh J, Wheeler JG, Hirschfield GM, et al. C-reactive protein and other circulating markers of inflammation in the prediction of coronary heart disease. $N$ Engl J Med. 2004;350(14): 1387-1397.

4. Blankenberg S, Tiret L, Bickel C, et al. Interleukin-18 is a strong predictor of cardiovascular death in stable and unstable angina. Circulation. 2002;106(1):24-30.

5. Ernst E. Fibrinogen: a "new" cardiovascular risk factor. Wien Med Wochenschr. 1991;141(17):375-382.

6. de Maat MP, Pietersma A, Kofflard M, Sluiter W, Kluft C. Association of plasma fibrinogen levels with coronary artery disease, smoking and inflammatory markers. Atherosclerosis. 1996; 121(2):185-191.

7. Danesh J, Lewington S, Thompson SG, et al. Plasma fibrinogen level and the risk of major cardiovascular diseases and nonvascular mortality: an individual participant meta-analysis. JAMA. 2005;294(14):1799-1809.

8. Ernst E. Plasma fibrinogen-an independent cardiovascular risk factor. J Intern Med. 1990;227(6):365-372.

9. Thompson SG, Kienast J, Pyke SD, Haverkate F, van de Loo JC. Hemostatic factors and the risk of myocardial infarction or sudden death in patients with angina pectoris. European Concerted Action on Thrombosis and Disabilities Angina Pectoris Study Group. N Engl J Med. 1995;332(10):635-641.

10. Espinola-Klein C, Rupprecht HJ, Bickel C, et al. Inflammation, atherosclerotic burden and cardiovascular prognosis. Atherosclerosis. 2007;195(2):e126-e134.

11. Kakafika AI, Liberopoulos EN, Mikhailidis DP. Fibrinogen: a predictor of vascular disease. Curr Pharm Des. 2007;13(16): 1647-1659.

12. Dejana E, Languino LR, Polentarutti N, et al. Interaction between fibrinogen and cultured endothelial cells. Induction of migration and specific binding. J Clin Invest. 1985;75(11):11-18.

13. Naito M, Hayashi T, Kuzuya M, Funaki C, Asai K, Kuzuya F. Effects of fibrinogen and fibrin on the migration of vascular smooth muscle cells in vitro. Atherosclerosis. 1990;83(1):9-14.
14. Tracy RP, Arnold AM, Ettinger W, Fried L, Meilahn E, Savage P. The relationship of fibrinogen and factors VII and VIII to incident cardiovascular disease and death in the elderly: results from the cardiovascular health study. Arterioscler Thromb Vasc Biol. 1999;19(7):1776-1783.

15. Kannel WB, Wolf PA, Castelli WP, D’Agostino RB. Fibrinogen and risk of cardiovascular disease. The Framingham Study. JAMA. 1987;258(9):1183-1186.

16. Whitney EJ, Krasuski RA, Personius BE, et al. A randomized trial of a strategy for increasing high-density lipoprotein cholesterol levels: effects on progression of coronary heart disease and clinical events. Ann Intern Med. 2005;142(2):95-104.

17. Chitolie A, Mackie IJ, Grant D, Hamilton JL, Machin SM. Inaccuracy of the 'derived' fibrinogen measurement. Blood Coagul Fibrinolysis. 1994;5(6):955-957.

18. Danesh J, Collins R, Appleby P, Peto R. Association of fibrinogen, C-reactive protein, albumin, or leukocyte count with coronary heart disease: meta-analyses of prospective studies. JAMA. 1998;279(18):1477-1482.

19. Green D, Foiles N, Chan C, Schreiner PJ, Liu K. Elevated fibrinogen levels and subsequent subclinical atherosclerosis: the CARDIA Study. Atherosclerosis. 2009;202(2):623-631.

20. Bolibar I, Kienast J, Thompson SG, Matthias R, Niessner H, Fechtrup C. Relation of fibrinogen to presence and severity of coronary artery disease is independent of other coexisting heart disease. The ECAT Angina Pectoris Study Group. Am Heart J. 1993;125(6):1601-1605.

21. Hartmann M, von Birgelen C, Mintz GS, et al. Relation between lipoprotein(a) and fibrinogen and serial intravascular ultrasound plaque progression in left main coronary arteries. J Am Coll Cardiol. 2006;48(3):446-452.

22. Ernst E, Koenig W. Fibrinogen and cardiovascular risk. Vasc Med. 1997;2(2):115-25.

23. Kockx M, de Maat MP, Knipscheer HC, et al. Effects of gemfibrozil and ciprofibrate on plasma levels of tissue-type plasminogen activator, plasminogen activator inhibitor-1 and fibrinogen in hyperlipidaemic patients. Thromb Haemost. 1997;78(4): 1167-1172.

24. Athyros VG, Kakafika AI, Karagiannis A, Mikhailidis DP. Do we need to consider inflammatory markers when we treat atherosclerotic disease? Atherosclerosis. 2008;200(1):1-12. 